\title{
Predictors of health care seeking for irritable bowel syndrome: a population based study
}

\author{
N J Talley, P M Boyce, M Jones
}

\begin{abstract}
Background-It has been suggested that psychological factors rather than symptoms drive subjects with irritable bowel syndrome (IBS) to seek medical care, but this issue has not been tackled in a population based study.

Aim-To identify whether psychological factors or abuse explain health care seeking for IBS.

Methods-A sample of residents of Penrith (a Sydney suburb representative of the Australian population) selected randomly from the electoral rolls (that by law include the entire population $\geqslant 18$ years) was mailed a validated self-report questionnaire. Measured were gastrointestinal symptoms including the Manning (and Rome) criteria for IBS, health care seeking, neuroticism (Eysenck Personality Questionnaire), psychological morbidity (General Health Questionnaire: GHQ) and sexual, physical and emotional abuse (including the standardised Drossman questions).
\end{abstract}

Results-Among 730 subjects, 96 (13\%, 95\% confidence interval (CI) 11-16\%) had IBS by the Manning criteria. Of those with IBS, $73 \%$ (95\% CI 63-81\%) had sought medical care for abdominal pain or discomfort. Only increasing pain severity $($ odds ratio $(O R)=2.10,95 \%$ CI 1.11-3.95) and duration of pain $(\mathrm{OR}=1.53,95 \% \mathrm{CI}$ 1.10-2.13) were independently associated with seeking health care for IBS. Pain severity was also predictive of recent care seeking (OR=1.74, 95\% CI 1.12-1.96). Neuroticism, psychological morbidity and abuse history were not significant predictors.

Conclusion-Psychological factors do not seem to explain health care seeking among community subjects with IBS.

(Gut 1997; 41: 394-398)

Keywords: epidemiology; irritable bowel syndrome; abuse; neuroticism

The irritable bowel syndrome (IBS) is a common and costly condition. ${ }^{12}$ Management of IBS remains unsatisfactory because the cause is obscure. ${ }^{3}$ Psychological factors and psychiatric disorders have been implicated in the pathogenesis but their role remains controversial. ${ }^{3-5}$

Two separate studies of volunteers have reported that psychological factors were associated with health care seeking by subjects with
IBS rather than IBS per se. ${ }^{67}$ Although not population based, these results suggest that psychological distress is not causally related to IBS but does drive subjects with IBS to seek care.$^{67} \mathrm{~A}$ history of abuse has been linked not only to $\mathrm{IBS}^{8-11}$ but also to health care seeking for bowel symptoms in one study. ${ }^{12}$ However, there are no careful population based studies, applying strict criteria for the identification of IBS, that have investigated the issue of whether psychological distress drives help-seeking behaviour among subjects with IBS.

We wished to test the hypothesis that psychological factors and abuse history account for health care seeking in IBS in the general population. We hypothesised that the personality trait neuroticism (characterised by an exaggerated response to physiological changes and physical symptoms in the absence of structural disease), ${ }^{13}$ psychological morbidity (anxiety or depression $)^{14}$ and childhood, but not adulthood, sexual abuse ${ }^{12}$ would be key predictors of visits to the doctor.

\section{Methods}

SUBJECTS

A total of 1500 subjects was randomly selected from the Electorate of Lindsay list of voters. ${ }^{15}$ This area, which includes Penrith and surrounding suburbs of Sydney, has a population of 73393 (3.6\% of the Sydney population) and is sociodemographically very similar to the Australian population according to 1991 Census data, except that its inhabitants are slightly younger and it has a slightly higher socioeconomic status. ${ }^{15} 16$ The list of selected subjects was divided into three batches of 500 subjects, and mailings of the bowel symptom questionnaire (BSQ) were conducted at intervals approximately two months apart, for ease of administration.

Subjects were offered a lottery ticket in return for completing the questionnaire. Subjects were given the option of refusing. Reminder letters were sent at two week intervals. At week 6 this included another questionnaire. At week 10, we attempted to telephone subjects who had not yet responded. Finally, the survey was closed at 12 weeks.

Of the 1500 people in the general community to whom we sent the questionnaire, 323 $(22 \%)$ had moved, and were excluded on that basis. Another 42 (3\%) were excluded due to serious illness, death, or language difficulties, leaving us with a valid sample of 1135 subjects receiving the questionnaire. By the close of the survey at the beginning of November 1995, $360(32 \%)$ subjects had explicitly refused to
Accepted for publication 20 February 1997 
participate. A completed questionnaire was returned by 730 subjects, giving a response rate of $64 \%$. Respondents were similar to the Australian population demographically but were slightly older $(69 \% \geqslant 40$ years compared with $61 \%$ in the Australian population).

QUESTIONNAIRE

The BSQ has been validated previously in Australia ${ }^{15}$ and is a modified version of the previously validated and widely used Bowel Disease Questionnaire. ${ }^{17}{ }^{18}$ It incorporates questions which identify the IBS. ${ }^{19}$ Visits to doctors and alternative therapists in the year before the survey and lifetime was also measured, as well as age, sex, marital status, and educational level.

We included in the BSQ two validated psychological questionnaires. The General Health Questionnaire (GHQ) was developed for population surveys. ${ }^{14}$ It was designed to detect non-psychotic psychiatric illness and is a state measure of psychiatric co-morbidity (assessing features in the previous few weeks); it does not provide a specific psychiatric diagnosis. The Eysenck Personality Questionnaire (10 item short neuroticism subscale) measures the personality trait neuroticism which we hypothesised would be important in promoting health care seeking. ${ }^{13}$

We included the sexual abuse questions validated by Drossman et $a l^{820}$ that we have applied in a US study of abuse and IBS, and these were not altered. ${ }^{9}{ }^{12}$ These questions were as follows:

- Has anyone ever exposed the sex organs of their body to you when you did not want it?

- Has anyone ever threatened to have sex with you when you did not want it?

- Has anyone ever touched the sex organs of your body when you did not want this?

- Has anyone ever forced you to have sex when you did not want this?

- Have you ever had any other unwanted sexual experiences not mentioned above?

The Drossman physical abuse questions in addition were included, with minor modifications. Changes in grammar and format were made to ensure comprehension by the local population. The referent terms used to identify the perpetrator in the questions relating to childhood abuse were changed to eliminate positive responses to minor events such as fights at school or bullying. Although it is conceded that a child could experience some long term consequences from such incidents if they were repetitive, frequent or severe, these events are usually qualitatively distinct from child abuse as it is generally understood. ${ }^{20}$ Consistent with this definition, any violent behaviour by older siblings would be considered "abuse", only if the sibling was in the position of being a care-giver for the child. This decision was adopted because in such a brief, self-report format it is not possible to discover the minority of cases where violence from older siblings or schoolmates occurred in circumstances sufficient to classify as abuse. Similarly, the change of wording for adult physical abuse was made to eliminate positive responses on the basis of one off events (e.g. mugging) or occasional violence from strangers or acquaintances (e.g. crime related or job related), as these events are outside the realm of abuse as conceptualised by our research definition.

Additionally, on the basis of our previous population based study ${ }^{12}$ we incorporated four new questions to assess emotional and psychological abuse. These questions have not yet been formally validated. However, they have excellent face validity, and represent a dimension of abuse which has been inadequately studied in relation to gastrointestinal disorders. Response options for the emotional and psychological abuse questions were identical to those used in the Drossman physical abuse questions (i.e. never, seldom, occasionally, or often). To increase the specificity of the survey, subjects endorsing physical or emotional abuse occurring at least often were considered to have suffered abuse.

\section{IRRITABLE BOWEL SYNDROME}

The Manning (and for comparison Rome) criteria were applied to identify subjects with IBS. By the Manning criteria, ${ }^{21}{ }^{22}$ as used in other population based studies, ${ }^{23}{ }^{24}$ IBS was defined as: abdominal pain that kept recurring over a period of more than three months in the previous year plus two or more of the following:

- looser stools at the onset of pain, often (more than $25 \%$ of the time);

- more frequent stools at the onset of pain, often;

- pain relief with defaecation, often;

- visible abdominal distension (defined as seeing the stomach or abdomen swell, or having to loosen a belt or clothes because of swelling), often;

- feelings of incomplete rectal evacuation, often;

- mucus per rectum.

IBS was also defined using the Rome criteria ${ }^{19}$ as more than three months of abdominal pain, in combination with one or more of the following symptoms:

- pain made better by bowel movement, often (more than $25 \%$ of the time);

- more and/or less bowel movements with pain, often;

- looser and/or harder stools with pain, often; and two or more of the following:

- more than three bowel movements daily, often and/or less than three bowel movements a week, often;

- stools very lumpy or hard, often and/or stools very loose or watery, often;

- incomplete rectal evacuation, often and/or urgency, often and/or straining, often;

- abdominal bloating or distension, often; and/or

- mucus in stools.

BOWEL HABIT SCORE

This was derived from counting the number of positive responses $(0-7)$ to the following questions: $>3$ stools per day, often; $<3$ stools per week, often; lumpy or hard stools, often; straining, often; looser or watery stools, often; urgency, often; and mucus per rectum. 
TABLE 1 A comparison between subjects with IBS by the Manning criteria who had and had not sought health care for abdominal pain or discomfort in the year before the survey

\begin{tabular}{lll}
\hline & $\begin{array}{c}\text { IBS health care seekers } \\
(n=70)\end{array}$ & $\begin{array}{l}\text { IBS non-health care } \\
\text { seekers }(n=26)\end{array}$ \\
\hline Mean (SD) age (y) & $46.6(15)$ & $43.9(14)$ \\
Female (\%) & 76 & 58 \\
Married or de facto (\%) & 77 & 73 \\
Pain severity (moderate or worse (\%)) & 83 & 73 \\
Pain once a week or more (\%) & 59 & 54 \\
Bowel habit score & $3.8(1.5)$ & $3.9(1.7)$ \\
Abdominal distension score & $1.7(0.6)$ & $1.4(0.8)$ \\
Neuroticism (mean (SD)) & $5.6(3.0)$ & $5.1(2.9)$ \\
Psychological morbidity (GHQ) (mean (SD)) & $3.5(3.5)$ & $2.8(3.7)$ \\
Completed high school (\%) & 36 & 46 \\
Childhood sexual abuse (\%) & 25 & 21 \\
Childhood rape (\%) & 2 & 43 \\
Childhood "other" sex experience (\%) & 9 & 16 \\
Adulthood sexual abuse (\%) & 28 & 12 \\
Adulthood rape (\%) & 17 & 4 \\
Adulthood "other" sex experience (\%) & 7 & 15 \\
Childhood physical abuse (\%) & 30 & 42 \\
Adulthood physical abuse (\%) & 31 \\
Childhood emotional abuse (\%) & 24 & 46 \\
Adulthood emotional abuse & 46 & 35 \\
Childhood abuse (any) (\%) & 33 &
\end{tabular}

$\mathrm{GHQ}=$ General Health Questionnaire.

DISTENSION SCORE

This was categorised as nil, bloating only (without visible distension) and visible abdominal distension.

\section{STATISTICAL ANALYSIS}

Several demographic variables were grouped into coarser categories because of small frequencies in some categories. Education was grouped as tertiary, high school diploma or greater (but not tertiary) and less than high school diploma. Marital status was grouped as currently married/de facto or not. For education and marital status these groups were considered appropriate as they reflected either milestones in education or the existence of a supportive relationship, respectively. Pain severity and time since onset of symptoms were left ungrouped but were treated as ordered discrete categories in the logistic regression analyses.

Four health care seeking variables were used as dependent variables: (1) visits to a doctor for any reason in the past 12 months; (2) visits to an alternate care provider for any reason in the past 12 months; (3) visits to a doctor or alternate care provider for abdominal pain or discomfort in the past 12 months; and (4) visits to a doctor or alternate care provider for abdominal pain or discomfort ever in the time period more than 12 months previously.

All variables were dichotomised. Having visited a doctor in the past 12 months for any reason or having visited a doctor or alternate therapist for abdominal pain or discomfort ever were dichotomised as rarely (never or occasionally) versus more than rarely (sometimes or often). The other care seeking indexes were categorised as never versus ever. The different categories reflect different distributions of response. The original intention was to categorise all four care seeking indexes as never versus ever. Inspection of the distribution of outcome revealed that for (1) and (4) fewer than $25 \%$ of subjects fell into the never category ( $4 \%$ for (1) and $22 \%$ for (4)). We considered that breaking such small numbers down by independent variables could lead to unreliable findings.

Statistical analysis involved three steps: description of variation in care seeking behaviour with all independent variables, univariate logistic regression of probability of care seeking on single independent variables and multivariate analysis using stepwise logistic regression. The last of these analyses sought evidence of statistically independent correlates of care seeking. The pseudo- $\mathrm{R}^{2}$ (deviance explained) is reported as a measure of the explanatory power of the multiple variable models.

All $\mathrm{p}$ values calculated were two tailed; the alpha level of significance was set at 0.05.

\section{Results}

HEALTH CARE SEEKING

Eighty nine per cent of subjects in the community (95\% confidence interval (CI) $87-91 \%$ ) had sought health care for any reason in the 12 months prior to the survey and $17 \%$ (95\% CI 14-20\%) had visited an alternative nonmedical health care provider in the previous year for any reason. Overall, 27\% (95\% CI $24-31 \%$ ) of the population had sought health care for abdominal pain or discomfort in the year prior to the study.

Ninety six subjects $(13 \%, 95 \%$ CI $11-16 \%)$ fulfilled the Manning criteria for IBS whereas 90 subjects $(12 \%, 95 \%$ CI $10-15 \%)$ fulfilled the Rome criteria for IBS. A substantial overlap in the subjects classified as IBS according to the two sets of criteria was observed; of the 102 classified as IBS by one or both definitions, 84 $(82 \%)$ were classified as IBS by both.

By the Manning criteria, 70 subjects with IBS had sought care for abdominal symptoms in the previous year $(73 \%, 95 \%$ CI $63-81 \%)$ and $78 \%$ with IBS had sought care at some time for symptoms (95\% CI 70-86\%). Most subjects with IBS by the Manning criteria (96\%, 95\% CI 90-99\%) had visited a doctor for some reason in the previous year while $27 \%$ (95\% CI 19-37\%) had sought alternative care. Similarly, a total of 65 subjects with IBS as defined by the Rome criteria reported having sought medical care for abdominal pain or discomfort in the previous year $(73 \%, 95 \%$ CI $63-82 \%$ ) and $79 \%$ had sought care at some time in the past for symptoms $(95 \%$ CI $69-87 \%)$.

PREDICTORS OF HEALTH CARE SEEKING

Table 1 summarises a comparison of the patient characteristics in subjects with IBS based on the Manning criteria who had and had not sought care for abdominal symptoms in the prior year. By stepwise logistic regression analysis, only pain severity was associated with a significantly increased probability of subjects with IBS having sought care for abdominal pain or discomfort within the previous 12 months (odds ratio $(\mathrm{OR})=1.74,95 \%$ CI $1.05-$ 2.88 ). This model explained just $4.4 \%$ of the deviance.

Of the potential correlates of the probability of subjects with IBS ever having sought care for abdominal symptoms, pain severity $(\mathrm{OR}=2.10$, 95\% CI 1.23-3.56), pain duration $(\mathrm{OR}=1.48$, 
95\% CI 1.12-1.96), visible distension ( $\mathrm{OR}=2.54,95 \%$ CI 1.10-5.83), and childhood sexual abuse (OR=2.89, 95\% CI 1.08-7.75) were significant. Of these, only pain severity $(\mathrm{OR}=2.10,95 \% \mathrm{CI} 1.11-3.95)$ and pain duration (OR $=1.53,95 \%$ CI 1.10-2.13) had statistically significant and independent effects on the probability of subjects with IBS ever having sought care for abdominal pain or discomfort in a final logistic model. This model explained $14.1 \%$ of the deviance.

A number of demographic, psychological and abuse characteristics were found to be associated with the probability of subjects with IBS (by the Manning criteria) seeking medical care for any reason within the past 12 months. These were advancing age $(\mathrm{OR}=1.05,95 \% \mathrm{CI}$ $1.01-1.08)$, pain severity $(\mathrm{OR}=1.79,95 \% \mathrm{CI}$ $1.10-2.89)$, pain duration $(\mathrm{OR}=1.35,95 \% \mathrm{CI}$ 1.04-1.75), current psychological morbidity ( $\mathrm{OR}=1.09,95 \%$ CI $1.01-1.17)$, visible distension $(\mathrm{OR}=4.06,95 \%$ CI 1.82-9.07), childhood "other" sexual abuse $(\mathrm{OR}=4.17,95 \%$ CI 1.12-15.57), adulthood emotional abuse $(\mathrm{OR}=3.13,95 \%$ CI 1.18-8.27), and adulthood "other" sexual abuse (OR=5.32, 95\% CI 1.13-25.12). Of these associations, only advancing age $(\mathrm{OR}=1.05,95 \%$ CI $1.00-1.10)$ and visible distension $(\mathrm{OR}=5.54,95 \% \mathrm{CI}$ 2.12-14.46) had statistically significant and independent effects on the probability of seeking medical care for any reason within the previous 12 months. This model explained $21.5 \%$ of the deviance.

As abdominal distension ended up in the final model rather than abuse for seeking medical care for any reason, we examined the relation between them. There were moderate correlations between the level of distension and the three abuse items (childhood "other", adulthood emotional, and adulthood "other") of univariate significance (Spearman rho 0.24, $0.29,0.20$, respectively). There was no evidence of effect modification by level of distension on the three abuse items from testing each interaction separately in logistic regression models.

No items showed evidence of being related to the probability of seeking care from an alternative care provider for any reason within the previous 12 months.

\section{Discussion}

We explored the link between psychological factors and health care seeking. We found that although psychological morbidity and abuse were associated with reports of seeing a doctor in some models, generally only symptoms were independent of significance. However, symptoms only explained a small amount of the behaviour in all the IBS models, suggesting that other unknown factors are much more important.

Predictors of health care seeking for those with IBS remain poorly defined. Symptom severity is well established to be important and our results confirm previous observations..$^{23}$ Heaton $e t$ al, in a population based study from the United Kingdom, observed that presentation for care in IBS correlated with the number of symptoms. ${ }^{25}$ They noted that abdominal pain headed the list in both sexes as a predictor of health care seeking, but psychological factors were not assessed. ${ }^{25}$

Psychological morbidity in the present study was only associated with seeking care for "any reason" and not for pain or discomfort, and was not significant at all in any of the final models. This suggests that psychological morbidity is not a predictor of health care seeking. Similarly, abuse was associated with health care seeking for any reason as well as abdominal symptoms ever but the specific type of abuse varied and was not significant in the final models, suggesting that the association was spurious. It is, however, noteworthy that "other" sexual abuse was unvariately associated with health care seeking for any reason by subjects with IBS. We do not know what these other unwanted sexual experiences were and so further work is needed to unravel this factor. The absence of childhood and adulthood abuse items from the final model testing health care seeking for any reason most likely reflects the correlation with distension and possibly the modest sample size. We did not measure stressful life events, social support networks or learned illness behaviour in childhood which may all be relevant, although studies of other illness have found that, even when these factors are accounted for, only a small amount of the variance in consultation rates is explained. ${ }^{26-28}$

One finding of note is that the majority of subjects with IBS in this population study had seen a doctor for gastrointestinal symptoms in the previous year in contrast to studies from the United States. ${ }^{23}{ }^{24}$ We found that over $70 \%$ of subjects with IBS in this study had sought care regardless of whether the Manning or Rome criteria were applied. This contrasts with population based data from the USA using a similar questionnaire where health care seeking was reported by one in four subjects with IBS using the Manning criteria. ${ }^{23}{ }^{24}$ However, our results are more comparable with findings from the United Kingdom, where Heaton and co-workers observed that the consultation rate was $75 \%$ in men and $76 \%$ in women with five or more IBS symptoms. ${ }^{25}$ The difference between United States and Australian consultation rates may reflect the health care system in Australia where universal free health care is available, which may encourage greater health care seeking. We also used a more rigorous definition of IBS in this study. In other research, transient symptoms could have been misclassified as IBS. ${ }^{21} 22$ In this study, subjects were required to have at least three months of pain and other chronic symptoms; this we suspect accounts for the slightly lower prevalence of IBS in this study (13\%) compared with previous reports of 15 to $20 \% .^{210}{ }^{23-25}$

The strengths of this study include the fact that it was population based. The responders were similar to the Australian population, suggesting that the sample was reasonably representative and hence the results are likely to be generally applicable. There is no reason to suppose that the questionnaire attracted a disproportionate number of complainers which 
could have biased the outcome measures. Furthermore, a validated set of symptom and psychological measures was applied. It is also reassuring that the Manning and Rome criteria identified similar subjects in the present study. However, a self-administered questionnaire can only obtain a limited amount of data and we had to rely on self-perceived symptoms and self-reports of health care seeking, which limit the interpretability of our results. Another limitation was the relatively small number of subjects with IBS who had not sought care available for analysis, reducing the power of the study.

We asked not only about help seeking in the previous year but also whether subjects had ever sought help, and we enquired about medical and non-medical consultation behaviour. We did not observe that consulters were more psychologically disturbed than non-consulters, in contrast to others. ${ }^{67}$ However, these US studies were based on volunteers rather than on a randomly selected population sample. We suspect that selection factors rather than differences between countries explain the contrasting results, but this remains to be confirmed. It is also unknown whether health care seeking for IBS varies according to ethnic groups in Australia. Zuckerman et al in the US have observed that Hispanics with IBS were less likely than non-Hispanic white subjects to have sought health care but were more likely to self-medicate. ${ }^{29}$ We did not assess ethnic status in the current study because this group would have comprised only a small proportion of the population ${ }^{15}$ but this issue needs to be explored further. We also did not measure what proportion in the sample had been referred to hospital or a specialist for assessment and whether those with IBS referred for specialist care differ from those not referred, but this important question needs to be tackled in future work.

In conclusion, neuroticism, psychological morbidity, and a history of abuse do not seem to explain health care seeking for IBS. Abdominal pain is an important predictor of help seeking but does not explain most of this behaviour. Whether patient fears including cancerophobia, different health care systems or other unrecognised factors can accurately predict help seeking in IBS have yet to be determined.

Supported in part by research grants from the University of Sydney (Bridging Grant) and the Royal Australasian College of Sydney (Bridging Grant) and the
Physicians (Cottrell Fellowship).

1 Talley NJ, Gabriel SE, Harmsen WS, Zinsmeister AR, Evans RW. Medical costs in community subjects with irritable RW. Medical costs in community subjects with irrita

2 Jones R, Lydeard S. Irritable bowel syndrome in the general population. BMF 1992; 304: 87-90.

3 Ford MJ. The irritable bowel syndrome. 7 Psychosom Med 1986; 30: 399-410.

4 Talley NJ, Phillips SF, Bruce B, Twomey CK, Zinsmeister AR, Melton LJ III. Relation among personality and symp- toms in nonulcer dyspepsia and the irritable bowel yndrome. Gastroenterology 1990; 99: 327-33.

5 Esler MD, Goulston KJ. Levels of anxiety in colonic disorders. N Engl f Med 1973; 288: 16-20.

6 Drossman DA, McKee DC, Sandler RS, Mitchell CM, Cramer EM, Lowman BC, et al. Psychosocial factors in the irritable bowel syndrome: A multivariate study of patients and nonpatients with irritable bowel syndrome. Gastroenterology 1988; 95: 701-8.

7 Whitehead WE, Bosmaiian L, Zonderman AB, Costa PT Jr, Schuster MM. Symptoms of psychologic distress associated with irritable bowel syndrome. Comparison of community and medical clinic samples. Gastroenterology 1988; 95: 709-14.

8 Drossman DA, Leserman J, Nachman G, Li Z, Gluck H, functional or organic gastrointestinal disorders. Ann Intern Med 1990; 113: 828-33.

9 Talley NJ, Fett SL, Zinsmeister AR. Self-reported abuse and gastrointestinal disease in outpatients: Association with irritable bowel-type symptoms. Am f Gastroenterol 1995; 90: $366-71$.

10 Longstreth GF, Wolde-Tsadik G. Irritable bowel symptoms in HMO examinees. Prevalence, demographic factors and clinical correlates. Dig Dis Sci 1993; 38: 1581-9.

11 Walker EA, Gelfand AN, Gelfand MD, Katon WJ. Psychiatric diagnoses, sexual and physical victimization, and disability in patients with irritable bowel syndrome or inflammatory bowel disease. Psychol Med 1995; 25: 1259-67.

12 Talley NJ, Helgeson SL, Zinsmeister AR, Melton LJ III. Gastrointestinal tract symptoms and self reported abuse: A population-based study. Gastroenterology 1994; 107: 1040-9.

13 Grayson DA. Latent trait analysis of the Eysenck Personality Questionnaire. F Psychiatry Res 1986; 20: 217-35.

14 Goldberg DP. The detection of psychiatric illness by questionnaire. London: Oxford University Press, 1972.

15 Talley NJ, Boyce P, Owen BK, Newman P, Paterson K. Initial validation of a bowel symptom questionnaire and measurement of chronic gastrointestinal symptoms in Australians. Aust NZ ₹ Med 1995; 25: 302-8.

16 Australian Bureau of Statistics. 1991 Census of population and housing, basic community profile: Area 6350 Penrith (C), ABS catalogue No. 2722.1.

17 Talley NJ, Phillips SF, Melton LJ III, Wiltgen C, Zinsmeister AR. A patient questionnaire to identify bowel disease. Ann Intern Med 1989; 111: 671-4.

18 Talley NJ, Phillips SF, Wiltgen CM, Zinsmeister AR, Melton LJ III. Assessment of functional gastrointestinal disease: The bowel disease questionnaire. Mayo Clin Proc 1990; 65: 1456-79.

19 Drossman DA, Richter JE, Talley NJ, Thompson WG, Corazziari E, Whitehead WE (eds). The functional gastrointestinal disorders. Boston: Little Brown, 1994.

20 Drossman DA, Talley NJ, Leserman J, Olden KJ, Barreiro $M$. Sexual and physical abuse and gastrointestinal illness: review and recommendations. Ann Intern Med 1995; 123: 782-94.

21 Manning AP, Thompson WG, Heaton KW, Morris AF. Toward positive diagnosis of irritable bowel. BMF 1978; 2: 653-4.

22 Talley NJ, Phillips SF, Melton LJ, Mulvihill C, Wiltgen C, Zinsmeister AR. Diagnostic value of the Manning criteria in irritable bowel syndrome. Gut 1990; 31: 77-81.

23 Talley NJ, Zinsmeister AR, Van Dyke C, Melton LJ III. Epidemiology of colonic symptoms and the irritable bowel syndrome. Gastroenterology 1991; 101: 927-34.

24 Talley NJ, Zinsmeister AR, Melton LJ III. Irritable bowel syndrome in a community: Symptom subgroups, risk factors and health care utilization. Am f Epidemiol 1995; 142: $76-83$.

25 Heaton KW, O'Donnell LJ, Braddon FE, Mountford RA, Hughes AO, Cripps PJ. Symptoms of irritable bowel syndrome in a British urban community: consulters and nonconsulters. Gastroenterology 1992; 102: 1962-7.

26 Verhaak PFM. Determinants of the help-seeking process: Goldberg and Huxley's first level and first filter. Psychol Med 1995; 25: 95-104.

27 Hunskaar S. One hundred and fifty men with urinary incontinence. II. Help seeking and self care. Scand F Prim Health Care 1992; 10: 26-9.

28 Phillips MA, Murrell SA. Impact of psychological and physical health, stressful events, and social support on subsequent mental health help seeking among older adults. $\mathcal{f}$ Consult Clin Psychol 1994; 62: 270-5.

29 Zuckerman MJ, Guerra LG, Drossman DA, Foland JA, Gregory GG. Health-care-seeking behaviour related to bowel complaints. Hispanic versus non-Hispanic whites. Dig Dis Sci 1996; 41: 77-82. 